\title{
Novel stable crystalline phase for the Stillinger-Weber potential
}

\author{
Flavio Romano, ${ }^{1,2, *}$ John Russo, ${ }^{1, \dagger}$ and Hajime Tanaka ${ }^{1, \ddagger}$ \\ ${ }^{1}$ Institute of Industrial Science, University of Tokyo, 4-6-1 Komaba, Meguro-ku, Tokyo 153-8505, Japan \\ ${ }^{2}$ Physical and Theoretical Chemistry Laboratory, Department of Chemistry, University of Oxford, \\ South Parks Road, Oxford, OX1 3QZ, United Kingdom
}

(Received 25 April 2014; revised manuscript received 8 July 2014; published 25 July 2014)

\begin{abstract}
Tetrahedral liquids such as silicon, germanium, carbon, water, and silica are an important class of materials not only for industrial applications but also for our understanding of nature. The Stillinger-Weber potential is one of the most popular models for computer simulations of these systems with tetrahedral coordination, with the directionality of the interactions introduced via a three-body repulsive term which promotes locally tetrahedral arrangements. This approach has been extended to various tetrahedral liquids, providing valuable insight into the physics of group XIV elements and more recently water. Perhaps surprisingly, a consistent thermodynamic picture of this class of models is still lacking despite their widespread usage. Here we fill this gap by computing equilibrium phase diagrams for the silicon and water parametrizations and report a novel crystal structure which dominates the models' phase diagram at intermediate and high pressure, and thus warrants further theoretical and numerical investigation. Our results redefine the phase behavior of an important class of tetrahedrally coordinated systems, and also suggest that a more stringent test for simulation models is the ability to select the experimentally relevant crystalline phases, as opposed to just reproducing their mechanical stability.
\end{abstract}

DOI: 10.1103/PhysRevB.90.014204

PACS number(s): 61.50.Ah, 61.66.Bi, 64.70.D-

\section{INTRODUCTION}

The Stillinger-Weber (SW) model is an iconic potential used in computer simulation to understand complex liquids with directional interactions. Originally proposed as an atomistic model for silicon [1], the SW model was shown to provide a good description of both the bulk diamond crystal [2] and the amorphous phases [3]. After the original parametrization, the SW potential has found widespread applicability for also modeling other group XIV elements, like germanium and carbon. The basic idea is that these elements, apart from energy and length scale differences, can be modeled by varying the relative strength of the three-body interaction used to impose local tetrahedrality, which becomes less strong with increasing atomic number.

The strength of the three-body interaction term was also shown to be the parameter that controls the glass-forming ability of this class of models [4]. By decreasing the relative importance of the three-body interaction with respect to the isotropic attraction, it has been found that the stable crystal phase at low pressure and temperature changes from the diamond cubic (dc), to the $\beta$-tin crystal, and finally to the body centered cubic (bcc). Importantly, as a function of the three-body strength, the melting temperature has a minimum where the dc, $\beta$-tin, and bcc structures compete for crystallization, and where the system is a fragile glass former. The analogy between this one-component eutectic point and the pressure behavior of group XIV elements has led to an experimental vitrification of metallic liquid germanium [5]. This V-shaped temperature-pressure phase diagram and the high glass-forming ability near the eutectic point are characteristic features of tetrahedral liquids, or water-type

\footnotetext{
*flavio.romano@gmail.com

†russoj@iis.u-tokyo.ac.jp

†tanaka@iis.u-tokyo.ac.jp
}

liquids [6,7]. In this respect, the SW model provides a clear example of the relationship between crystallization and glass forming ability $[6,8]$ in a context where the thermodynamic behavior is well understood.

In recent years, potentials of the SW class have gained popularity as coarse-grained models for complex liquids. A recent successful example is the $\mathrm{mW}$ model for water, which represents the water molecule as a monoatomic SW-like particle with a three-body interaction strength intermediate between that of silicon and carbon [9]. The model reproduces with surprising accuracy the structure and anomalies of liquid water at a fraction of the computational cost, and, differently from atomistic models of water, can be easily crystallized $[10,11]$. The SW potential has also been used to model reduced-valence systems as a way to achieve equilibrium gels [12], similarly to approaches based on patchy interactions [13-15].

The SW potential plays a central role in topics as wide as semiconductor bulk properties, water phase behavior, and crystallization pathway, and the physics of arrested states, both glasses and gels, in the presence of directional interactions. Despite its importance, the phase diagram of this class of potentials has not been thoroughly determined. For silicon, thermodynamic calculations have focused on the low-pressure melting line of the diamond crystal [16-19]. There is a surprising lack of thermodynamic data at intermediate and high pressure, where silicon is expected to undergo a dc to $\beta$-tin transition [20-23]. Also for $\mathrm{mW}$ water most calculations focused only on the dc and dh (diamond hexagonal) phases $[10,24]$. Zero pressure melting calculations for different values of the three-body interaction strength were performed by melting of small clusters, and thus are likely to give a qualitatively correct picture but possibly suffer from finite-size effects [4]. Moreover, the tetrahedral nature of the SW potential is compatible with countless crystal structures, and new metastable phases are constantly being discovered [25-27], and it is important to assess their thermodynamic role. 
In this work we systematically derive the equilibrium phase diagrams for the SW potential with special focus for the parametrizations for silicon and $\mathrm{mW}$ water. We selected the candidate crystal structures from an extended list of lattices that are known either from experiment or numerical predictions for a collection of tetrahedral systems. Specifically, we try all the ice forms [28], the stable and metastable phases of silicon, silica (quartz), and all group XIV elements, as well as other locally tetrahedral structures. We will show that the stable phase for SW silicon and $\mathrm{mW}$ water at high pressure is not the $\beta$-tin phase, as previously thought, but a novel crystal which is named here sc16, with a simple cubic unit cell and 16 atoms per unit cell.

\section{METHODS}

\section{A. Numerical methods}

In order to compute the phase diagrams we run Monte Carlo simulations in the the canonical $N V T$ or isothermalisobaric NPT ensembles. Trial moves in the former cases are standard translation attempts, where the average size of the translation attempt is adjusted at every state point with short preproduction runs to yield a success ratio of roughly 0.4. Isothermal-isobaric simulations of the liquid phase also involve standard volume-change attempts, on average 1 every $N$ translation attempts, where a small change in length scale is attempted. Again, the average size of the volume move attempt is automatically adjusted in short preproduction runs to yield a success ratio of roughly 0.2 . Importantly, we run isothermalisobaric simulations of solid phases, allowing fluctuations in the box shape, i.e., allowing anisotropic responses to the pressure [29,30]. As pointed out in Ref. [31], this is crucial unless the solid phases is known to be cubic, which is the case only for two of the studied structures.

All the systems are composed of approximately $N \approx 1000$ particles, a size large enough so that finite-size effects are negligible.

We compute free energies and coexistence lines as outlined in Ref. [31]. For the liquid phase, we use the Widom insertion method [32] to compute the excess free energy of a liquid state at relatively high temperature. This method is easy to implement, but as discussed Ref. [33] it is very slow at converging at low $T$. We have found that, in all the models studied, the Widom method is very reliable for $T>0.25$, allowing uncertainties in the (excess) free energy of the liquid of the order of $0.001 k_{\mathrm{B}} T$ per molecule within a few hours of simulation time. Once the free energy of the liquid phase is known at fixed $N, T$, and $V$, it is possible to use thermodynamic integration (again, see Ref. [31]) to compute the free energy in any state point where equilibration is attainable.

The procedure to compute the free energy of a solid phase is a little more complicated, although nowadays quite well established. We use the Frenkel-Ladd procedure [33]. The SW model is well suited for this technique, and it allows us to achieve a relatively high accuracy, again of the order of $0.001 k_{\mathrm{B}} T$ per molecule. We point out that the three-body potential does not require any special treatment.

To estimate the accuracy of our results, we have checked the coexistence point obtained with two-phase direct coexistence
[34], where simulations are started with a box that is half crystalline and half liquid in the isothermal-isobaric ensemble. Several simulations are run for the same value of $P$ and different values of $T$ close to the predicted coexistence temperature $T_{\text {coex }}$ from free-energy calculations. If $T>T_{\text {coex }}$ the solid half of the box will melt, otherwise it will grow. This technique is quite accurate, although if the difference in free energy between the two phases is small it is possible that either the interface is slow at growing/melting or that simulations for the same values of $P$ and $T$ can sometimes melt the crystal and sometimes grow it. That is the origin of the error bars in Fig. 3. Technically, one should keep the component of the pressure perpendicular to the surface fixed and set the other components to zero, but as discussed in Ref. [31] this hardly makes any difference.

Hamiltonian integration is also employed to study the melting line at zero pressure for different strengths of the three-body interaction term.

\section{B. The generalized SW potential}

The SW potential [1] is composed of the sum of two-body and three-body terms, with a dimensionless parameter $\lambda$ that controls their relative strength:

$$
U=\sum_{i} \sum_{j>i} U_{2}\left(\mathbf{r}_{i j}\right)+\lambda \sum_{i} \sum_{j \neq i} \sum_{k>j} U_{3}\left(\mathbf{r}_{i j}, \mathbf{r}_{i k}\right) .
$$

The two-body term comprises a steep repulsion at very short separations and a short-range attraction:

$$
U_{2}(r)=A \epsilon\left[B\left(\frac{\sigma}{r}\right)^{p}-\left(\frac{\sigma}{r}\right)^{q}\right] \exp \left(\frac{\sigma}{r-a \sigma}\right)
$$

the three-body term is a directional repulsion which promotes specific conformations in between triplets of particles, modeling the effect of directional interactions:

$$
\begin{aligned}
U_{3}\left(r_{i j}, r_{i k}\right)= & \epsilon\left[\cos \theta_{i j k}-\cos \theta_{0}\right]^{2} \\
& \times \exp \left(\frac{\gamma \sigma}{r_{i j}-a \sigma}\right) \exp \left(\frac{\gamma \sigma}{r_{i k}-a \sigma}\right) .
\end{aligned}
$$

The common parameters for the models considered in this work are $A=7.049556277, B=0.6022245584, p=4$, $q=0, \cos \theta_{0}=-1 / 3$. These are the parameters introduced originally by Stillinger and Weber [1], with $\theta_{0}$ enforcing the tetrahedrality of the interactions. The parameter $\epsilon$ sets the energy scale, while $\sigma$ sets the length scale. For silicon, $\epsilon=50.003 \mathrm{kcal} / \mathrm{mol}$ and $\sigma=2.0951 \AA$. For $\mathrm{mW}$ water, $\epsilon=$ $6.189 \mathrm{kcal} / \mathrm{mol}$ and $\sigma=2.3925 \AA$. In order to easily compare the different parametrizations, we will use reduced units where $\epsilon$ and $\sigma$ are the units of energy and length, respectively. The only parameter that differentiates the models is thus the strength of the three-body term $\lambda$, which is set to $\lambda=21$ for silicon [1] and $\lambda=23.15$ for water [9]. For convenience, we provide here the conversions factors between the internal units of temperature ([T]) and pressure ([p]) for both silicon and $\mathrm{mW}$ water. $[T]_{\text {silicon }}=25157 \mathrm{~K},[p]_{\text {silicon }}=377674$ bars, $[T]_{\mathrm{mW}}=3114.4 \mathrm{~K}$, and $[p]_{\mathrm{mW}}=31400$ bars. 
TABLE I. Crystalline structures considered in this work, divided into three categories: (i) unstable, mechanically unstable crystals for the SW potential; (ii) unfavorable, crystal structures with high internal energies; and (iii) candidate, structures with low internal energies for which we conduct full free energy calculations. For each crystalline structure we report the results from the energy minimization procedure. Where binary crystals are considered, only the component with local tetrahedral symmetry is considered.

\begin{tabular}{|c|c|}
\hline Crystal & Note \\
\hline Cubic diamond, Si I, dc & candidate \\
\hline Hexagonal diamond, Si IV, dh & candidate \\
\hline bc8, Si III [35] & transforms into sc 16 \\
\hline st12, Ge III & candidate \\
\hline bcc & at high $\lambda$ melts into sc \\
\hline fcc, Si X & very high pressures \\
\hline bct5 [36] & candidate \\
\hline$\beta$-tin, Si II [35] & candidate \\
\hline ice-II & unfavorable \\
\hline ice-III & equivalent to st12 \\
\hline ice-IV & unstable \\
\hline ice-V & transforms in $\beta$-tin \\
\hline ice-i and ice-i' [37] & unfavorable \\
\hline ice-VI & unstable \\
\hline ice-VII & unstable \\
\hline imma, Si XI [25] & unstable \\
\hline Ibam $[26]$ & unfavorable \\
\hline Simple hexagonal, Si V [35] & unfavorable \\
\hline Cmca, Si VI [38] & unfavorable \\
\hline $\mathrm{t} 12[27]$ & candidate \\
\hline R8, Si XII [39] & transforms into sc16 \\
\hline bt 8 & transforms into sc16 \\
\hline Simple cubic, sc & unfavorable \\
\hline Clathrate, Si34 [40] & candidate \\
\hline Clathrate, Si46 & candidate \\
\hline Clathrate, Si136 & candidate \\
\hline Pyrite & candidate \\
\hline Marchesyte & unfavorable \\
\hline alpha cristobalite & unfavorable \\
\hline alpha low & unfavorable \\
\hline alpha $\mathrm{N}_{2}$ & unfavorable \\
\hline
\end{tabular}

\section{Crystalline structures}

We systematically investigated over 20 different crystalline structures, chosen from the stable and metastable crystalline states of silicon, water, and crystalline structures of other materials with tetrahedral symmetry. The full list of crystalline structures considered is reported in Table I. For each crystalline structure we have conducted a steepest-descent energy minimization procedure for different values of the density and for different values of the unit cell and crystal parameters. Some crystals are not mechanically stable in the SW model, as they either transform into other crystals during the energy minimization procedure or form a disordered configuration. Other crystals, while being mechanically stable, have internal energies higher than the liquid state at all densities and were thus discarded as high free-energy structures. We point out that all the stable crystals have average energy per particle $u$ very close to the ground state energy $u=-2 \epsilon$. For all the crystal
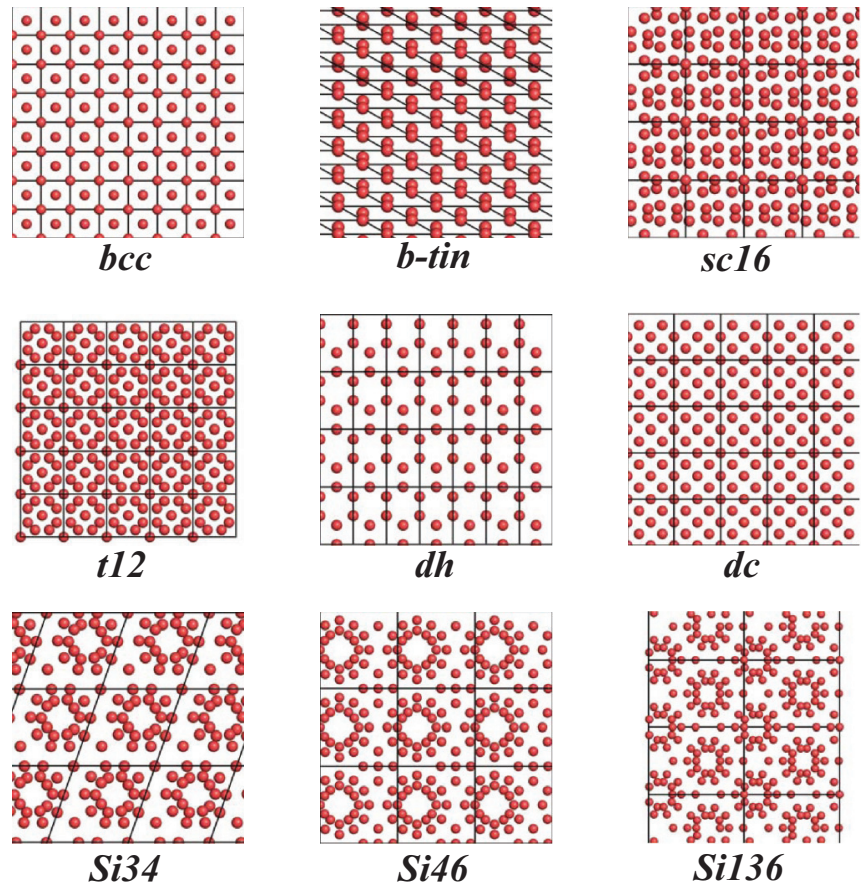

FIG. 1. (Color online) Low-free-energy crystal structures for the $\mathrm{SW}$ and $\mathrm{mW}$ potentials. The first row shows the structures relevant at high pressures (above the upper triple point): bcc, $\beta$-tin, and sc 16 . The second row shows the structures at intermediate pressures (in between the triple points): $\mathrm{t} 12$, dh, and dc. The third row depicts the clathrate phases, i.e., the crystals relevant at negative pressures: Si34, $\mathrm{Si} 46$, and $\mathrm{Si} 136$. For each crystal the unit cells are bounded by black lines.

structures that have energy lower than that of the liquid, we performed full free-energy calculations in order to identify the stable ones and compute the coexistence lines.

\section{RESULTS AND DISCUSSION}

In Fig. 1 we show the thermodynamically relevant crystal structures for silicon and $\mathrm{mW}$ water. At high pressure (above the upper triple point), the stable crystals are bcc, $\beta$-tin, and sc16 at low, intermediate, and high strengths of the three-body interaction, respectively. The relevant crystalline structures at intermediate pressures (in between the two triple points) are the $\mathrm{t} 12, \mathrm{dc}$, and dh crystals. At negative pressures, the relevant crystalline structures are the low-density clathrate phases, Si34, Si46, and Si136.

\section{A. The sc16 crystal}

One of the main results of this work is the discovery of the novel sc16 crystal phase, which is the stable phase for these models at high pressure. The formation of this phase was observed during simulations of the bc 8 crystal, which spontaneously transforms into the sc16 structure at intermediate and high pressure. The sc16 crystal structure has the same point symmetry as the bc8 crystal, but has a simple cubic unit cell, instead of the body-centered unit cell of the bc 8 structure. The transition pathway between the two crystals is shown in Fig. 2, where each pair of atoms with separation 


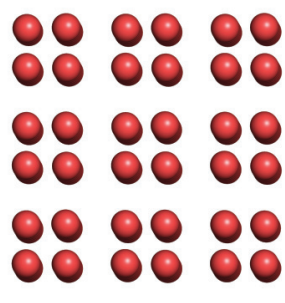

bc8

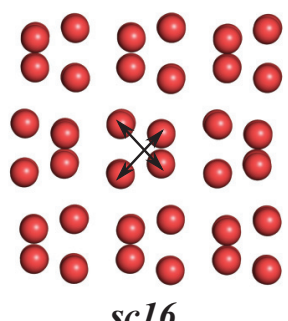

sc16
FIG. 2. (Color online) bc8 and sc16 crystal structures. The transformation path between the two crystals is indicated by the arrows.

$(1 / 2,1 / 2,1 / 2)$ in the original bc 8 structure is shifted by the same amount along the separation vector (see the arrows in Fig. 2). The space group of the sc16 crystal is $P a \overline{3}$, where two atoms occupy the Wickoff position $8 c$, with parameters $x_{1}$ and $x_{2}$, respectively. We provide here the crystal structure parameters for the state point $P=0.135$ and $T=0.05$ in internal units. The lattice parameter is $a=3.14$, and the atoms positions in the primitive cell are $x_{1}=0.328$ and $x_{2}=0.120$.

For convenience, in Table II we provide real space coordinates of the 16 atoms in the unit cell, which can be replicated along the three axis to obtain a crystal of the desired size.

\section{B. Phase diagram of SW silicon}

The thermodynamic information we obtained is reported in Fig. 3. We start the discussion with the phase diagram of the original parametrization for silicon: The stable phases are expected to be the clathrate phases at negative pressures, the diamond cubic $(\mathrm{dc})$ at low but positive pressures, and $\beta$-tin at intermediate to high pressures [20,22]. We point out that the hexagonal diamond phase $(\mathrm{dh})$ is thermodynamically equivalent to dc for this model, as already shown for $\mathrm{mW}$ [41] and other short-range potentials [42]. The phase diagram is shown in Fig. 3(a). The results agree with previous calculations

TABLE II. Unit cell of the sc16 crystal for the $\mathrm{mW}$ potential at $T=0.05$ and $P=0.135$. The unit cell has size $L_{x}=3.14, L_{y}=$ $3.14, L_{z}=3.14$.

\begin{tabular}{lcc}
\hline \hline$x$ & $y$ & $z$ \\
\hline 1.03 & 1.03 & 1.03 \\
2.60 & 0.54 & 2.11 \\
2.60 & 1.03 & 0.54 \\
1.03 & 0.54 & 2.60 \\
0.54 & 2.11 & 2.61 \\
2.11 & 2.60 & 0.54 \\
2.11 & 2.11 & 2.11 \\
0.54 & 2.60 & 1.03 \\
0.38 & 0.38 & 0.38 \\
1.95 & 1.20 & 2.77 \\
1.95 & 0.38 & 1.19 \\
0.37 & 1.19 & 1.95 \\
1.19 & 2.76 & 1.95 \\
2.76 & 1.95 & 1.19 \\
2.76 & 2.77 & 2.77 \\
1.19 & 1.95 & 0.38 \\
\hline \hline
\end{tabular}

for negative and low pressures, and we attribute the small differences to finite-size effects in the previously reported results. At high pressure, instead, we find a new stable crystal for the SW potential, the sc16 crystal, which preempts the $\beta$-tin structure. For comparison with earlier results [22], we also report the virtual melting line of the $\beta$-Si phase and its coexistence line with the dc and dh crystals [see the green symbols in Fig. 3(a)]. We also note that this model is a crystal former, and that the crystal which is spontaneously nucleated is expected to be a random stacking of the dc and dh crystals. Which nucleates first and what is the ratio of cubic to hexagonal can depend on kinetic effects which are not reflected in the thermodynamics.

\section{Phase diagram of $\mathbf{m W}$ water}

We next show that the sc 16 crystal structure is also the stable crystalline phase for $\mathrm{mW}$ water at high pressures. Apart from dimensional units, the only difference with SW silicon is in the strength of the three-body interaction, $\lambda=23.15(\lambda=21$ in silicon). Overall, $\mathrm{mW}$ water is just a slight modification of the silicon potential, and it is reasonable to expect a very similar equilibrium picture for the two models. Indeed, this is the case for the phase diagram reported in Fig. 3(b). The stable crystalline phases of $\mathrm{mW}$ water are the same as the ones of silicon: clathrates at negative pressures, hexagonal and cubic ice (equivalent to $\mathrm{dh}$ and $\mathrm{dc}$ ) at intermediate pressures, and the sc16 crystal at high pressures. The melting temperatures are higher, signaling that the liquid structure changes with the increased directionality of the interaction, a point which has been addressed very recently [43].

Comparing the phase diagram of the two models [Figs. 3(a) and $3(\mathrm{~b})]$, one can see that the stability domain of the crystals increases from going from silicon to water: The coexistence lines are located at higher $T$ and the triple point liquid/ice $I_{h} / \mathrm{sc} 16$ moves to higher $P$. This provides a possible explanation of the better crystal-forming ability of $\mathrm{mW}$ with respect to SW: The higher melting temperature of the $\mathrm{dc} / \mathrm{dh}$ crystals allows significant supercooling at temperatures where the dynamics is still fast. There is a difference in the pressure dependence of the melting line of the dc/dh crystals, which is stronger for silicon and weaker for water. Again, since the crystal structure is virtually unaffected by the change of model, this reflects changes in the liquid structure. Interestingly, a metastable phase at low pressure for both models is the $t 12$ crystal structure, originally proposed as an allotrope for group XIV elements [27]. We recently found that this metastable crystalline structure plays a crucial role in the homogeneous nucleation of ice, in line with Ostwald's step rule of phases [41].

Our results show that the $\mathrm{mW}$ model, while it has been shown to provide a good representation of water's behavior at low pressures, it is not a robust model of water at high pressure where it fully retains the behavior of the silicon model from which it was derived. We should note that all high-pressure ice polymorphs have a high free energy in this model, and some are not even mechanically stable, since they are not local minima for the potential energy. This suggests that to reproduce the complex high-pressure phase diagram of 

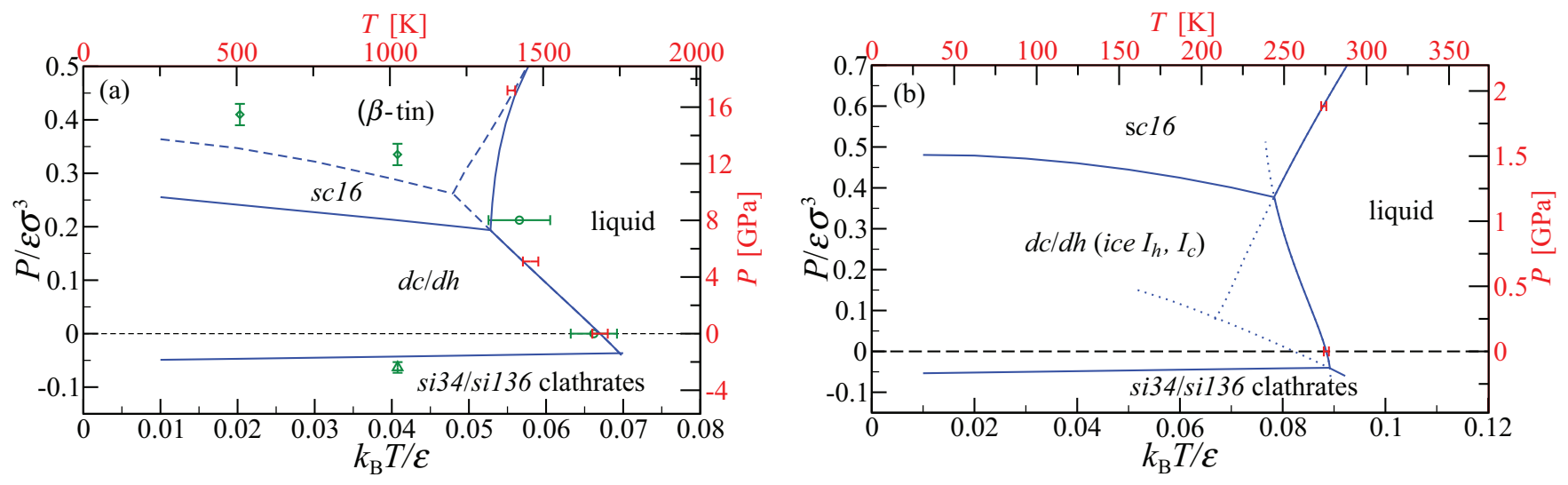

FIG. 3. (Color online) Equilibrium phase diagrams for the SW model in the silicon (a) and mW water (b) parametrization. In both cases, the stable crystals at negative pressure are the Si34 and Si136 clathrates, which have the same free energy within our resolution. The Si46 clathrate is metastable. As expected, the low-pressure stable phases are the dc and dh phases (ice $I_{c}$ and $I_{h}$ in water nomenclature), again thermodynamically equivalent for this class of models. The high pressure phase is the sc16 phase reported in this work. In (a) we also report with dashed lines the metastable coexistence lines between the $\beta$-tin phase, which was thought to be the most stable phase, and dc and the liquid phases. The red bars show coexistence points between crystals and liquid that that have been tested with direct coexistence simulations precision, and the length of the bars shows the confidence interval. The green symbols and bars in (a) are the results of [22], where sc16 was not reported. Dotted lines in (b) are the metastable continuation of the corresponding coexistence line.

water a representation that includes the hydrogens is probably unavoidable.

\section{Phase diagram as a function of $\lambda$}

A further point worth investigating is the phase diagram of the SW model class at $P=0$ with varying $\lambda$, reported in Fig. 4 for $\lambda \in[15,24]$. The previous phase diagrams show that decreasing $\lambda$, going from water model to silicon, stabilizes the fluid phase with melting lines shifting to lower temperatures. The triple point also moves at lower pressures, so that the high pressure phases gain stability with decreasing $\lambda$. We thus expect the diamond cubic phase to progressively lose stability, eventually becoming metastable for low enough values of $\lambda$. This was addressed in Ref. [4], where it was shown that the melting temperature, at zero pressure, has a minimum with

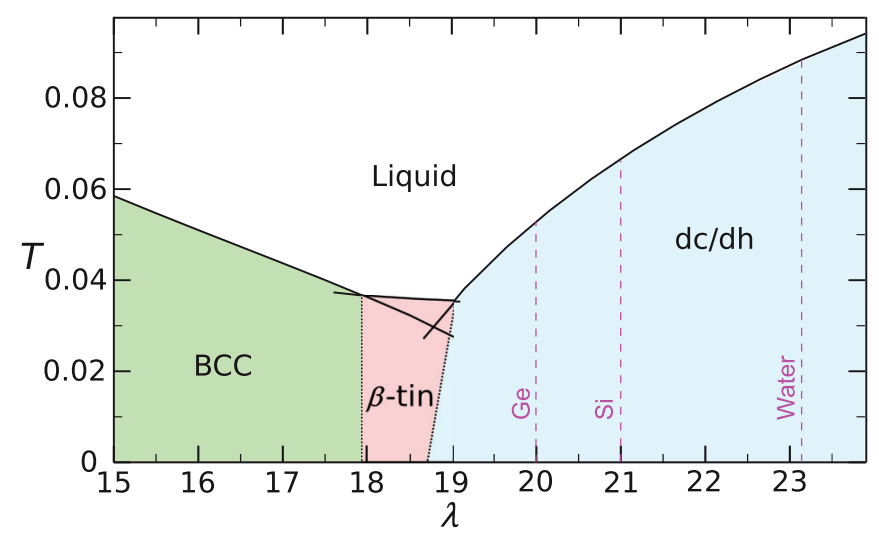

FIG. 4. (Color online) Zero pressure phase diagram as a function of the tetrahedrality parameter $\lambda$. The continuous (black) lines are the melting lines for the bcc, $\beta$-tin, and dc phases (respectively at low, intermediate, and high values of $\lambda$ ). Vertical dashed lines indicate the value of $\lambda$ in models for germanium, silicon, and $\mathrm{mW}$ water. decreasing $\lambda$. This minimum is found when the stable phase is the $\beta$-tin phase. It was observed that this minimum signals a region of high glass-forming ability. At high values of $\lambda$ the stable phases at $P=0$ are both the dc and dh phases, as we already showed for $\mathrm{mW}$ water and silicon. Decreasing $\lambda$ stabilizes the liquid phase, with a progressive lowering of the melting temperature, until a new stable phase, the $\beta$-tin crystal, appears. With decreasing the strength of the threebody interaction, the high pressure phases extend to lower pressure, and in this regime the $\beta$-tin crystal becomes stable with respect to the sc16 crystal. The stability domain of the $\beta$-tin phase is small, extending in the interval $18 \lesssim \lambda \lesssim 19$. At lower $\lambda$ the stable phase becomes the bcc phase, and the melting temperature steeply increases with decreasing $\lambda$.

\section{CONCLUSION}

In summary, we have computed the equilibrium phase diagram of the SW and $\mathrm{mW}$ models from full free-energy calculations. Despite their importance, a consistent thermodynamic picture was missing for both of these models. We have also reported a new crystalline structure, sc16, which is the thermodynamically stable crystal at high pressure for both SW and $\mathrm{mW}$. Testing whether this structure can be experimentally relevant for some tetrahedral material or whether it is compatible with a more detailed description of silicon or water would be an interesting avenue of research. Regarding the $\mathrm{mW}$ model, we should stress that while it is successful at ambient pressure, its predictions should be taken with care at higher pressures since its equilibrium behavior diverges substantially from what has been found with experiment and more detailed water models. We should stress that the major technical difficulties in assessing the phase diagrams of tetrahedral liquids lies in the large number of possible stable crystalline phases that have to be considered. A possible approach is to use special algorithms [29,30,44] to identify crystal candidates and then study their thermodynamic 
properties. In this work we instead consider naturally occurring crystalline structures, previously identified in the literature, and systematically investigate their potential energy landscape. This allowed us to identify a relatively small number of candidate crystalline structures for which full free energy calculations were conducted. For both silicon and water we confirm the presence of stable open crystal structures, the dc and dh phases at low pressures, and the clathrate phases at negative pressures. The new crystalline phase sc16 preempts the expected $\beta$-tin phase at intermediate and high pressures. Despite the large number of crystalline phases which were not taken into consideration in previous studies, our results support the picture of the zero pressure phase diagram of the SW model as a function of $\lambda$ [4], which displays an eutectic point corresponding to a $\beta$-tin phase at intermediate values of $\lambda$. Finally, our results suggest that a stringent test for a simulation model is to check whether it is able not only to reproduce the experimentally relevant crystals, but also to select the right structures as the thermodynamically stable ones. Our work also sheds light on which features of the interaction potential are responsible for the selection of a particular crystal structure of tetrahedral liquids.

\section{ACKNOWLEDGMENTS}

F. Romano and J. Russo contributed equally to this work. This study was partly supported by Grants-in-Aid for Scientific Research (S) and Specially Promoted Research from the Japan Society for the Promotion of Science (JSPS), the Aihara Project, the FIRST program from JSPS, initiated by the Council for Science and Technology Policy (CSTP), and a JSPS short-term fellowship for F.R.
[1] F. H. Stillinger and T. A. Weber, Phys. Rev. B 31, 5262 (1985).

[2] H. Balamane, T. Halicioglu, and W. A. Tiller, Phys. Rev. B 46, 2250 (1992).

[3] V. V. Vasisht, S. Saw, and S. Sastry, Nat. Phys. 7, 549 (2011).

[4] V. Molinero, S. Sastry, and C. A. Angell, Phys. Rev. Lett. 97, 075701 (2006).

[5] M. H. Bhat, V. Molinero, E. Soignard, V. C. Solomon, S. Sastry, J. L. Yarger, and C. A. Angell, Nature (London) 448, 787 (2007).

[6] H. Tanaka, Eur. Phys. J. E 35, 113 (2012).

[7] H. Tanaka, Phys. Rev. B 66, 064202 (2002).

[8] H. Shintani and H. Tanaka, Nat. Phys. 2, 200 (2006).

[9] V. Molinero and E. B. Moore, J. Phys. Chem. B 113, 4008 (2008).

[10] E. Moore and V. Molinero, Nature (London) 479, 506 (2011).

[11] A. Reinhardt and J. P. Doye, J. Chem. Phys. 136, 054501 (2012).

[12] S. Saw, N. L. Ellegaard, W. Kob, and S. Sastry, J. Chem. Phys. 134, 164506 (2011).

[13] E. Zaccarelli, J. Phys.: Condens. Matter 19, 323101 (2007).

[14] J. Russo, P. Tartaglia, and F. Sciortino, J. Chem. Phys. 131, 014504 (2009).

[15] L. Rovigatti and F. Sciortino, Mol. Phys. 109, 2889 (2011).

[16] J. Q. Broughton and X. P. Li, Phys. Rev. B 35, 9120 (1987).

[17] S. Yoo, X. C. Zeng, and J. R. Morris, J. Chem. Phys. 120, 1654 (2004).

[18] K.-C. Fang and C.-I. Weng, Nanotechnology 16, 250 (2005).

[19] V. Dozhdikov, A. Y. Basharin, and P. Levashov, J. Chem. Phys. 137, 054502 (2012).

[20] P. F. McMillan, Nat. Mater. 1, 19 (2002).

[21] C. C. Yang, J. C. Li, and Q. Jiang, Solid State Commun. 129, 437 (2004).

[22] M. Kaczmarski, O. N. Bedoya-Martinez, and E. R. Hernández, Phys. Rev. Lett. 94, 095701 (2005).

[23] J. Behler, R. Martoňák, D. Donadio, and M. Parrinello, Phys. Rev. Lett. 100, 185501 (2008).

[24] D. T. Limmer and D. Chandler, J. Chem. Phys. 135, 134503 (2011).

[25] A. Mujica, A. Rubio, A. Munoz, and R. J. Needs, Rev. Mod. Phys. 75, 863 (2003).
[26] B. D. Malone and M. L. Cohen, Phys. Rev. B 85, 024116 (2012).

[27] Z. Zhao, F. Tian, X. Dong, Q. Li, Q. Wang, H. Wang, X. Zhong, B. Xu, D. Yu, J. He et al., JACS 134, 12362 (2012).

[28] M. Chaplin, http://www1.lsbu.ac.uk/water/ice.html.

[29] L. Filion, M. Marechal, B. van Oorschot, D. Pelt, F. Smallenburg, and M. Dijkstra, Phys. Rev. Lett. 103, 188302 (2009).

[30] J. de Graaf, L. Filion, M. Marechal, R. van Roij, and M. Dijkstra, J. Chem. Phys. 137, 214101 (2012).

[31] C. Vega, E. Sanz, J. L. F. Abascal, and E. G. Noya, J. Phys.: Condens. Matter 20, 153101 (2008).

[32] B. Widom, J. Chem. Phys. 39, 2808 (1963).

[33] D. Frenkel and B. Smit, Understanding Molecular Simulation: From Algorithms to Applications (Academic, New York, 2001), Vol. 1.

[34] A. J. C. Ladd and L. V. Woodcock, Chem. Phys. Lett. 51, 155 (1977).

[35] J. Z. Hu, L. D. Merkle, C. S. Menoni, and I. L. Spain, Phys. Rev. B 34, 4679 (1986).

[36] L. L. Boyer, E. Kaxiras, J. L. Feldman, J. Q. Broughton, and M. J. Mehl, Phys. Rev. Lett. 67, 715 (1991).

[37] C. J. Fennell and J. D. Gezelter, J. Chem. Theory Comput. 1, 662 (2005).

[38] M. Hanfland, U. Schwarz, K. Syassen, and K. Takemura, Phys. Rev. Lett. 82, 1197 (1999).

[39] J. Crain, G. J. Ackland, J. R. Maclean, R. O. Piltz, P. D. Hatton, and G. S. Pawley, Phys. Rev. B 50, 13043 (1994).

[40] A. San-Miguel, P. Kéghélian, X. Blase, P. Mélinon, A. Perez, J. P. Itié, A. Polian, E. Reny, C. Cros, and M. Pouchard, Phys. Rev. Lett. 83, 5290 (1999).

[41] J. Russo, F. Romano, and H. Tanaka, Nat. Mater. 13, 733 (2014).

[42] F. Romano, E. Sanz, and F. Sciortino, J. Chem. Phys. 132, 184501 (2010).

[43] M. Singh, D. Dhabal, A. H. Nguyen, V. Molinero, and C. Chakravarty, Phys. Rev. Lett. 112, 147801 (2014).

[44] M. A. Zwijnenburg, K. E. Jelfs, and S. T. Bromley, Phys. Chem. Chem. Phys. 12, 8505 (2010). 